\title{
Síntomas depresivos posinfarto al miocardio: detección precoz en una población hospitalizada
}

\author{
Bernardita Blümel $\mathrm{M}^{\mathrm{a}}$, Allister Gibbons Fa, Andrés Kanacri Ca, \\ Nicolás Kerrigan $B^{a}$, Ramón Florenzano U.
}

\section{Depressive symptoms after an acute myocardial infarction}

Background: Depression after myocardial infarction (MI) is a frequent disorder and it increases the long-term risk of cardiac mortality. Aim: To assess the frequency of depressive symptoms and the history of depression in hospitalized post-MI patients. Patients and Methods: During three months, depressive symptoms and history of depression were studied in 47 consecutive patients (mean age 59,8 $\pm 9,5$ years, $68 \%$ male), admitted for MI to the Barros Luco Trudeau Hospital. The Beck Depression Inventory (BDI) and the Composite International Diagnostic Interview (CIDI 2.1) were used with DSM-IV diagnosis criteria. Results: According to the results obtained using the CIDI, 27,7\% of the patients had a history of depression. This occurred in $53,3 \%$ of women and $15,6 \%$ of men $(p<0.01)$. During the hospitalization, $38,3 \%$ of patients had depressive symptoms (BDI $\geq 17$ points), affecting $60 \%$ of women and $28,1 \%$ of men $(p<0.02)$. In women and patients with history of depression, depressive symptoms tended to be more common and more severe. Conclusions: Depressive symptoms in post-MI patients are frequent and attending physicians should actively detect them (Rev Méd Chile 2005; 133: 1021-27).

(Key Words: Depression, Heart diseases; Myocardial infarction)

Recibido el 1 de diciembre, 2004. Aceptado el 19 de mayo, 2005.

Servicio de Psiquiatría, Hospital del Salvador. Santiago, Chile.

anterno de Medicina, Universidad de los Andes. Santiago, Chile.

L a enfermedad cardiovascular es la principal causa de muerte en los países desarrollados y presenta, a su vez, un aumento progresivo en los países en vías de desarrollo ${ }^{1,2}$. Esta situación ha generado la necesidad de diseñar estrategias preventivas, especialmente orientadas a reducir los factores modificables de riesgo cardiovascular,

Correspondencia a: Srta. Bernardita Blümel M. El Ciruelillo 4000, Dpto 904 - Vitacura, Santiago de Chile. Teléfono: 3217571. E mail: berniblumel@yahoo.es vinculados estrechamente con las condiciones de vida del mundo moderno que deterioran la calidad de vida ${ }^{3,4}$.

Por otra parte, se ha señalado a la depresión como el trastorno mental característico de la segunda mitad del siglo XX. En Santiago de Chile, la prevalencia de vida de depresión en 1994, alcanzó $11,3 \%$ en población general ${ }^{5}$. Un estudio realizado por Florenzano et al, en la ciudad de Santiago, encontró una prevalencia de depresión en pacientes que consultan en el nivel primario de atención de $29,5 \%{ }^{6}$. 
Recientemente la depresión ha sido considerada una patología ligada a enfermedades cardiovasculares y no sólo un factor de riesgo para enfermedad coronaria; es una patología de alta prevalencia en pacientes que han sobrevivido a un infarto del miocardio (IM) ${ }^{7}$. Dentro de la evidencia disponible, Ziegelstein ha señalado que aproximadamente uno de cada seis pacientes experimenta depresión mayor luego de un IM, y 1 de cada 3 presentan síntomas depresivos durante la hospitalización ${ }^{8}$. Otra investigación en pacientes pos IM, demostró que 17,2\% presentó síntomas leves o moderados de depresión, y 15,2\% depresión 0 distimia ${ }^{9}$. Un estudio de seguimiento de Lesperance et al, determinó que $31,5 \%$ de los pacientes infartados experimentó depresión durante la hospitalización y el primer año posterior al alta. Del total de pacientes con depresión, 50\% la presentó durante la hospitalización, 42,9\% entre el alta y 6 meses, y 7,1\% entre 6 y 12 meses después del IM. Por otra parte, aquellos pacientes que tenían el antecedente de depresión, tuvieron un riesgo mayor de sufrir un nuevo episodio de depresión pos $\mathrm{IM}$, tanto en el hospital como después del alta ${ }^{10}$.

En los últimos años, se ha reconocido la importancia de la depresión en la evolución de los pacientes pos IM: constituye un factor de riesgo independiente que incrementa el riesgo de mortalidad, tanto en hombres como en mujeres, durante al menos los primeros 18 meses siguientes al evento coronario ${ }^{11-13}$. Lauzon et al, demostraron que existía una diferencia estadísticamente significativa en la mortalidad a un año de ocurrido el IM, entre aquellos pacientes que presentaban depresión al momento de la hospitalización y aquéllos sin depresión (16\% v/s 8\% respectivamente) ${ }^{14}$.

Aún no han sido identificados los mecanismos precisos por los cuales la depresión aumenta el riesgo de mortalidad en pacientes pos IM. Sin embargo, existen diversas hipótesis que podrían explicar esta relación, entre las cuales se señala: 1) aumento de la activación y agregación plaquetaria, 2) aumento del tono adrenérgico que predispone a arritmias ventriculares, 3) activación del sistema inmune, implicado tanto en el desarrollo de la placa aterosclerótica como en el aumento de la mortalidad de pacientes pos IM, 4) menor adherencia al tratamiento farmacológico y adopción de estilos de vida saludable y 5) menor soporte social ${ }^{15}$.
La coexistencia de algún trastorno psiquiátrico puede complicar el diagnóstico, la evolución y el tratamiento de cualquier patología médica. A su vez, son mayores los porcentajes de discapacidad y de costos de atención ${ }^{16,17}$. Por ello es importante estudiar la frecuencia de depresión entre los pacientes hospitalizados. La literatura internacional comunica alta frecuencia de depresión en pacientes hospitalizados, que va entre $17 \%$ y $35,5 \%{ }^{18-20}$. En nuestro país, una cifra menor fue encontrada por Hernández $\mathrm{G}$ et al, en los pacientes del Servicio de Medicina Interna del Hospital Barros Luco Trudeau, en donde detectaron una prevalencia de $9,8 \%{ }^{20}$. A pesar de que la evidencia muestra una alta frecuencia de depresión asociada a IM y un impacto negativo sobre la evolución de la enfermedad coronaria, es poco frecuente su pesquisa y controvertido su tratamiento $^{12}$. Por otra parte, en Chile no existen estudios que hayan evaluado la presencia de depresión en pacientes hospitalizados posterior a un evento coronario. Sólo existe un trabajo realizado por Araya et al, que evaluó la presencia de depresión mayor como factor de riesgo en la enfermedad coronaria en nuestro país ${ }^{21}$.

Este trabajo tiene como objetivo estudiar la relación entre depresión y enfermedad coronaria en pacientes hospitalizados en un hospital del Servicio de Salud Metropolitano Sur de Santiago de Chile. Los objetivos específicos son:

1. Establecer la frecuencia de síntomas depresivos en pacientes ingresados por IM.

2. Establecer el antecedente de depresión en pacientes hospitalizados por IM.

\section{Pacientes y MÉTOdos}

Esta investigación corresponde a un estudio transversal y descriptivo. Se seleccionaron todos aquellos pacientes con el diagnóstico de IM admitidos en forma consecutiva en el Hospital Barros Luco Trudeau (Servicio de Salud Metropolitano Sur), desde el 8 de septiembre al 8 de diciembre del año 2003. Los criterios de inclusión fueron: pacientes con diagnóstico de IM, basado en criterios clínicos, electrocardiográficos y enzimáticos, y libre voluntad para participar en el estudio para lo cual firmaron un consentimiento informado. Los criterios de exclusión fueron los siguientes: pa- 
cientes en riesgo vital, compromiso cuantitativo y cualitativo de conciencia, deterioro cognitivo, hipoacusia severa, incapacidad de comunicarse, falta de consentimiento o alta hospitalaria temprana (menor a cinco días de hospitalización). De un total de 53 pacientes que cumplieron con los requisitos para ingresar al estudio, 47 dieron su consentimiento en forma voluntaria, ellos constituyeron los sujetos de este estudio.

Se realizó una entrevista, donde los autores solicitaron a los pacientes que respondieran verbalmente una encuesta socio-demográfica y la escala de depresión del Composite International Diagnostic Interview (Lifetime CIDI 2.1) de la Organización Mundial de la Salud ${ }^{22}$, mientras que el Inventario de Depresión de Beck (Beck Depresion Inventory, BDI) fue completado por los propios pacientes ${ }^{23}$. Todos los instrumentos fueron analizados por los autores, quienes habían sido capacitados por un equipo docente con experiencia en los instrumentos antes citados. A partir de esta entrevista se obtuvieron datos demográficos, médicos y psicosociales, que incluyeron: edad, sexo, estado civil, años de educación, ocupación, factores de riesgo de enfermedad coronaria, diagnósticos médicos previos y antecedentes psiquiátricos. El BDI, en su adaptación castellana para Latinoamérica, fue utilizado para pesquisar síntomas depresivos actuales, a contar del momento del IM. Un puntaje de 17 o más se considera positivo para síntomas depresivos, según describe el propio autor ${ }^{24}$. Se empleó esta herramienta estandarizada con el objeto de poder comparar resultados con otros estudios internacionales y por ser un instrumento autoadministrable. El Lifetime CIDI, versión validada en Chile, fue utilizado para determinar episodios depresivos pasados y actuales, con criterios del DSM-IV ${ }^{25}$. Los datos médicos fueron obtenidos a partir de la revisión de fichas clínicas, donde se incluyeron alteraciones electrocardiográficas, alza de enzimas cardíacas, clasificación de Killip y tratamientos recibidos (angioplastia, trombólisis, terapia antiagregante y cirugía de bypass coronario).

Estadística. Los datos obtenidos fueron tabulados, codificados y analizados con el programa computacional SPSS 11.0 (SPSS Inc. Septiembre 2001). Las pruebas estadísticas utilizadas fueron: Prueba exacta de Fisher, $\mathrm{Chi}^{2}$, prueba Z (Mann-Whitney) y t de Student según correspondiera.

\section{RESULTADOS}

Las encuestas fueron realizadas, en promedio, a los 7,1 $\pm 2,1(\mathrm{x} \pm \mathrm{DE})$ días de hospitalización, con un máximo de 12 días y un mínimo de 5 días. La muestra estuvo constituida por 47 pacientes; los hombres representaron $68,1 \%$ y las mujeres $31,9 \%$. La edad promedio fue de $59,8 \pm 9,5$ años y la escolaridad del grupo fue de $9,6 \pm 4,8$ años. Con respecto al estado civil, predominaron los pacientes casados $(72,3 \%)$, seguidos por los viudos $(12,8 \%)$, los separados $(10,6 \%)$ y por último los solteros (4,3\%). El 46,8\% de los pacientes estaba laboralmente activo.

Las características clínicas de la muestra se exponen en la Tabla 1. Cabe destacar, que 3 pacientes $(6,4 \%)$ refinieron un diagnóstico psiquiátrico previo, que en todos los casos fue depresión. Dentro de los antecedentes mórbidos de la muestra, destaca que 8 pacientes habían tenido un IM antiguo.

Resultados CIDI. Los resultados obtenidos con el Lifetime CIDI 2.1 se aprecian en la Tabla 2. Sólo 3

\section{Tabla 1. C aracterísticas clínicas de 47 pacientes hospitalizados por IM en el H ospital Barros Luco Trudeau}

\begin{tabular}{|lrc|}
\hline & $\mathrm{n}$ & $\%$ \\
\hline Antecedentes mórbidos & 34 & 72,3 \\
Antecedentes psiquiátricos & 6 & 12,8 \\
Factores de riesgo coronario & 44 & 93,6 \\
$\quad$ Diabetes Mellitus 2 & 12 & 25,5 \\
Hipertensión arterial & 24 & 51,1 \\
Tabaquismo & 30 & 63,8 \\
Dislipidemia & 21 & 44,7 \\
Clase Killip & & \\
I & 36 & 76,6 \\
II & 9 & 19,1 \\
III & 1 & 2,1 \\
IV & 1 & 2,1 \\
Tratamiento & & \\
Angioplastía & 0 & 0 \\
Cirugía de by-pass coronario & 0 & 0 \\
Trombólisis & 22 & 46,8 \\
Antiagregante & 25 & 53,2 \\
\hline
\end{tabular}



Tabla 2. Resultados del BD I y del CID I según sexo y antecedente de depresión (CID I)
de 47 pacientes hospitalizados por IM en el H ospital Barros L uco Trudeau

\begin{tabular}{|lccccc|}
\hline & $\begin{array}{c}\text { Total } \\
(\mathrm{n}=47)\end{array}$ & $\begin{array}{c}\text { Hombres } \\
(\mathrm{n}=32)\end{array}$ & $\begin{array}{c}\text { Mujeres } \\
(\mathrm{n}=15)\end{array}$ & $\begin{array}{c}\text { Con antecedentes } \\
(\mathrm{n}=13)\end{array}$ & $\begin{array}{c}\text { Sin antecedentes } \\
(\mathrm{n}=34)\end{array}$ \\
$\begin{array}{l}\text { Depresión previa } \\
\text { según CIDI (\%) }\end{array}$ & 27,7 & 15,6 & $53,3^{(1)}$ & 100 & 0 \\
\hline BDI & & & & & \\
Promedio $\pm \mathrm{DE}$ & $16,9 \pm 10,9$ & $14,8 \pm 8,8$ & $21,3 \pm 13,8$ & $23,3 \pm 14,3$ & $14,4 \pm 8,3^{(3)}$ \\
$\begin{array}{l}\text { Mediana } \\
\geq 17(\%)\end{array}$ & 14 & 14 & 18 & 19 & 14 \\
& 38,3 & 28,1 & $60^{(2)}$ & 61,5 & $29,4^{(4)}$ \\
\hline
\end{tabular}

${ }^{(1)} \mathrm{Chi}^{2} \mathrm{p}<0,01 ;{ }^{(2)} \mathrm{Chi}^{2} \mathrm{p}<0,02$; ${ }^{(3)}$ Prueba t $\mathrm{p}<0,05$; ${ }^{(4)} \mathrm{Chi}^{2} \mathrm{p}<0,07$

$(23,1 \%)$ de los 13 pacientes que presentaron depresión a lo largo de su vida entregaron el antecedente de una enfermedad psiquiátrica previa. Tres pacientes $(6,4 \%)$ se encontraban cursando un episodio depresivo al momento de la entrevista. Excluyendo a éstos, ningún otro paciente había presentado un episodio depresivo durante el último año.

Resultados BDI. En la Tabla 2 se pueden observar los resultados obtenidos con el BDI. La Tabla 3 muestra el porcentaje de pacientes con síntomas depresivos agrupados según gravedad.

Como se observa en la Tabla 2, los pacientes con antecedentes de depresión en algún momento de su vida, en relación con aquéllos que no la han presentado, tienden a tener un mayor riesgo de presentar síntomas depresivos pos IM (OR 3,35; IC 95\%, 0,89-12,63). Asimismo, las mujeres tendrían mayor riesgo que los hombres de presentar síntomas depresivos pos IM (OR 5,1; IC 95\%, 1,36-19,16).

No se encontraron diferencias estadísticamente significativas para presencia de síntomas depresivos, ni puntajes del BDI más altos, al comparar los grupos por edad, estado civil, la clase Killip, escolaridad y pacientes laboralmente activos en comparación con aquellos que no se encontraban trabajando al momento del IM.

De los 18 pacientes con síntomas depresivos, desde el día de la hospitalización hasta el momento en que se realizó la entrevista, solamente uno

Tabla 3. Clasificación de síntomas depresivos según gravedad en 47 pacientes hospitalizados por IM en el H ospital Barros Luco Trudeau

\begin{tabular}{|ccrr|}
\hline Puntaje BDI & Síntomas depresivos & N & $\%$ \\
\hline $0-10$ & Normales & 14 & 29,8 \\
$11-16$ & Leve alteración del ánimo & 15 & 31,9 \\
$17-20$ & Leves & 4 & 8,5 \\
$21-30$ & Moderados & 9 & 19,1 \\
$31-40$ & Graves & 3 & 6,4 \\
$>40$ & Severos & 2 & 4,3 \\
\hline
\end{tabular}


(5,6\%) había sido visitado por el equipo de salud mental a solicitud de su médico tratante.

\section{DisCUSIÓN}

El 27,7\% de los pacientes presentaron depresión en algún momento de su vida, siendo más frecuente en las mujeres (OR 6,17; IC 95\%, 1,5324,84). A pesar de que diversos estudios sugieren que la presencia de depresión en la población general sería un factor de riesgo para la enfermedad coronaria ${ }^{8,23}$, la presencia de depresión durante el año previo al infarto, en nuestro estudio, solamente ocurrió en $6,4 \%$ de los pacientes. Araya et al, señalaron una prevalencia de depresión mayor de 28,6\% durante los últimos 6 meses al $\mathrm{IM}^{21}$. Existen diferencias metodológicas y demográficas entre ambos estudios: zonas geográficas (Iquique), herramientas diagnósticas (DSM III-R) y la entrevista fue realizada en el domicilio de los pacientes entre 6 y 24 meses posterior al alta, hechos que hacen difícil la comparación.

La presencia de síntomas depresivos es frecuente en el paciente hospitalizado por un IM, afectando aproximadamente a dos de cada cinco pacientes $(38,3 \%)$. Sin embargo, esta cifra difiere de la prevalencia de depresión comunicada en 1 de cada 10 pacientes $(9,8 \%)$, por otro estudio realizado en el Servicio de Medicina Interna del mismo centro hospitalario ${ }^{20}$. Esta diferencia podría ser explicada porque la enfermedad coronaria aguda suele ser frecuentemente percibida como un evento con riesgo vital, implica cambios en el estilo de vida y a la vez son pacientes sometidos a cuidados y tratamientos más agresivos en relación a aquellos hospitalizados en un servicio de medicina interna.

Los síntomas depresivos (BDI $\geq 17$ puntos) tienden a ser más graves y más frecuentes en los pacientes que presentan depresión en algún momento de su vida y en mujeres. Estos antecedentes podrían ser factores predictores para presentar síntomas depresivos pos IM. Aparentemente, no influiría la edad, el estado civil, clase Killip, ni el hecho de estar trabajando al momento del evento coronario, en la aparición de síntomas depresivos en estos pacientes.

Nuestros resultados difieren al ser comparados con trabajos internacionales, a pesar de no em- plear la misma metodología. Diversas publicaciones norteamericanas señalan que la incidencia de síntomas depresivos utilizando el BDI, en pacientes hospitalizados por un IM, sería entre 17,2\% y $32 \% 11,27$. Estos estudios utilizaron un punto de corte inferior al nuestro para considerar la presencia de síntomas depresivos (BDI $\geq 10$ puntos). Utilizando este punto de corte, el porcentaje de pacientes con síntomas depresivos en nuestro caso se elevaría a 70,2\%. Una posible explicación para esta diferencia sería la mayor prevalencia de depresión en nuestro medio ${ }^{5,6}$, las diferencias de ambiente hospitalario y el tratamiento recibido para el IM.

A pesar de la alta frecuencia de depresión en pacientes pos IM y de su impacto negativo sobre la evolución de la enfermedad coronaria, en la práctica clínica actual es infrecuente encontrar como parte de los cuidados habituales de estos pacientes las evaluaciones de la presencia de depresión. Existe un plan nacional de tratamiento de la depresión de eficacia comprobada ${ }^{26}$. La depresión por sí sola justificaría la necesidad de tratamiento, ya que afecta gravemente la calidad de vida. Se ha sugerido que los inhibidores selectivos de la recaptación de serotonina (ISRS), y en particular la sertralina, tendrían efectos favorables sobre la disfunción plaquetaria en los pacientes con depresión ${ }^{27,28}$.

Es importante considerar que existen limitaciones en nuestro estudio, como por ejemplo, el hecho de haber utilizado herramientas que no nos permitieron hacer el diagnóstico intrahospitalario de depresión. Además, el número total de pacientes evaluados no permitió establecer categóricamente algunos resultados estadísticamente significativos. Finalmente, al utilizar un sólo centro hospitalario, no es posible extrapolar los resultados a toda la población chilena.

Sería de interés, como se ha realizado en otros estudios ${ }^{11,27}$, hacer un seguimiento de estos pacientes, para evaluar la persistencia, gravedad y aparición de síntomas depresivos en los meses posteriores al alta, así como la mortalidad a largo plazo. Eventualmente se podría evaluar si una intervención terapéutica, sea ésta farmacológica 0 no, tendría algún impacto a nivel de la evolución posterior a un evento isquémico.

En conclusión los síntomas depresivos pos IM durante la hospitalización son frecuentes en la 
práctica clínica, y deben ser pesquisados precozmente por el médico tratante, con el fin de mejorar la calidad de vida y posiblemente la sobrevida. A su vez, también es importante identificar a los pacien-

\section{REFERENCIAS}

1. Sytowski $\mathrm{P}$, Kannel W, D'Agostino R. Changes in Risk Factors and Decline in Mortality from Cardiovascular Diseases: the Framingham Heart Study. N Engl J Med 1990; 32: 1635-41.

2. Danber T, Meadows G, Moore F. Epidemiological Approaches to Heart Disease: The Framingham Study. Am J Pub Health 1951; 41: 279-86.

3. Berríos X. Tendencia temporal de los factores de riesgo de enfermedades crónicas: ¿La antesala silenciosa de una epidemia que viene? Rev Méd Chile 1997; 125: 1405-7.

4. Jadué L, Vega J, Escobar M, Delgado I, Garrido C, Lastra P ET AL. Factores de riesgo para las enfermedades no transmisibles. Metodología y resultados globales de la encuesta base del programa CARMEN. Rev Méd Chile 1999; 127: 1004-13.

5. Rioseco P, Escobar B, Vicente B, Vielma M, Saldivia $\mathrm{S}$, CRUZAT P et al. Prevalencia de vida de algunos trastornos psiquiátricos en la provincia de Santiago. Rev Psiquiatría 1994; 4: 186-93.

6. Florenzano R, acuña J, Fulerton C, Castro C. Estudio comparativo de frecuencia y características de los trastornos emocionales que consultan en el nivel primario de atención en Santiago de Chile. Rev Méd Chile 1998; 126: 397-405.

7. Muselman D, Evans D, Nemeroff C. The relationship of depression to cardiovascular disease: Epidemiology, biology, and treatment. Arch Gen Psychiatry 1998; 55: 580-92.

8. Ziegeistein R. Depression in patients recovering from a Myocardial Infarction. JAMA 2001; 286: 1621-7.

9. Ziegeistein R. Depression after myocardial infarction. Cardiol Rev 2001; 9: 45-51.

10. Lesperance F, Frasure-Smith N, Talajic M. Major depression before and after myocardial infarction: its nature and consequences. Psychosom Med 1996; 58: 99-110. tes con mayor riesgo de presentar síntomas depresivos después del evento coronario, entre los cuales destacan el grupo de mujeres y aquéllos que tienen el antecedente previo de depresión.

11. Luutonen S, Holm H, Salminen J, Risla A, Salokangas R. Inadequate treatment of depression after myocardial infarction. Acta Psychiatr Scand 2002; 106: 434-9.

12. Lesperance F, Frasure-Smith N, Juneau M, Theroux P. Depression and 1-year prognosis in unstable angina. Arch Intern Med 2000; 160: 1354-60.

13. Frasure-Smith N, Lesperance F, Juneau M, Talajic M, BouRASSA MG. Gender, depression, and one-year prognosis after myocardial infarction. Psychosom Med 1999; 61: 26-37.

14. Lauzon C, Beck C, Huynh T, Dion D. Depression and prognosis following hospital admission because of acute myocardial infarction. CMAJ 2003; 168: 547-52.

15. Ziegelstein R. Patients with depression are less likely to follow recommendations to reduce cardiac risk during recovery from a myocardial infarction. Arch Intern Med 2000; 160: 1818-23.

16. LEVENSON J, HaMer R, Rossiter L. Relation of psychopathology in general medical inpatients to use and cost of services. Am J Psychiatry 1990; 147: 1498-503.

17. Hansen MS, Fink P, Frydenberg M, Oxhoj M-L, SONDERGAARD L, ERIKSEN M. Mental disorders in medical inpatients and the association to severity of illness, self-rated physical disability, health perception. Psychosomatics 2001; 42: 41-7.

18. Ly Kouras E, Ioannidis $C$, Voulgari A, Jemos J, Tzonou A. Depression Among General Hospital Patients in Greece. Acta Psychiatr Scand 1989; 79: 148-52.

19. Stewart M, Drake F, Winokur G. Depression Among Medically ill Patient. Gen Hosp Psychiatry 1982; 4: 171-8.

20. Hernández G, IbáÑez C, Kimelman M. Prevalencia de trastornos psiquiátricos en hombres y mujeres hospitalizados en un Servicio de Medicina Interna de un hospital de Santiago de Chile. Rev Méd Chile 2001; 129: 1279-88.

21. Araya M, Bustos C, Madariaga C, Tomé M. La depresión como nuevo factor de riesgo en la 
cardiopatía coronaria en Chile. Rev Méd Chile 2002; 130: 1249-56.

22. Vielma M, Vicente B, Rioseco P, Castro N, Torres S. Validación en Chile de la entrevista diagnóstica estandarizada para estudios epidemiológicos CIDI. Rev Psiquiatría 1992; 4: 1039-49.

23. Conde C, Useros E. Adaptación castellana de la escala de evaluación conductual para la depresión de Beck. Rev Psiquiat Psicol Med 1975; 12: 217-36.

24. Beck A, Steer R, Garbin M. Psychometric properties of the Beck Depression Inventory: twenty five years of evaluation. Clin Psychol Rev 1998; 8: 77-100.

25. Diagnostic and Statistical Manual of Mental Disorders (DSMIV). Task force on Diagnosis and Nomenclature. American Psychiatric Association. American Psychiatric Press, Washington DC, 1994.

26. Minoletti A, López C, Brockering W, Flores L, George M, JimÉnez P. Personas afectadas por depresión. Plan Nacional de Salud Mental y Psiquiatría.
Ministerio de Salud, Gobierno de Chile. Segunda Edición 2001. Disponible en: http:// www.minsal.cl/ici/s 1/u 14/Depresión.pdf. [Consultado el 14 de enero de 2004].

27. Van den Brink R, van Meil J, Honig A, Schene A, CRjJns H, Lambert F et al. Treatment of depression after myocardial infarction and the effects on cardiac prognosis and quality of life: Rationale and outline of the Myocardial Infanction and Depression-Intervention Trial (MIND-IT). Am Heart J 2002; 144: 219-25.

28. Markovitz J, Shuster J, Chitwood W. Platelet activation in depression and effects of sertraline treatment: an open-label study. Am J Psychiatry 2000; 157: 1006-8.

29. Writing Committee for the ENRICHD Investigators. Effects of Treating Depression and Low Perceived Social Support on Clinical Events After Myocardial Infarction. The Enhancing Recovery in Coronary Heart Disease Patients (ENRICHD) Randomized Trial. JAMA 2003; 289: 3106-16.

Agradecimientos

Agradecemos la colaboración de: Dr. Jorge Bartolucci J, Dr. Rubén Lamich B, Dr. Ricardo Larrea G. y en particular al Dr. César Carvajal A. 